\title{
Zustandsüberwachung des tribomechanischen Systems Massezylinder/Plastifizierschnecke in der Kunststoffverarbeitung
}

\author{
Bernhard Praher, Georg Steinbichler \\ Johannes Kepler Universität Linz, Institute of Polymer Injection Moulding and Process Automation, \\ Altenberger Strasse 69, 4040 Linz, Österreich
}

\begin{abstract}
Kurzfassung
Aufgrund der hohen Temperaturen und Drücken in der Kunststoffverarbeitung gibt es bis heute keine effiziente Möglichkeit den Verschleiß verschiedener Komponenten von Plastifiziereinheiten (eingesetzt beim Spritzgießen und der Extrusion) während des Betriebes zu messen. Zwischen dem Schneckensteg der rotierenden Plastifizierschnecke und der Massezylinderinnenwand ist ein Spalt (ca. $100 \mu \mathrm{m}$ ) vorhanden. In diesem Spalt befindet sich aufgeschmolzene Kunststoffschmelze, die den direkten Kontakt der beiden Komponenten verhindern soll. Durch verschiedene Verschleißmechanismen (Abrasion, Adhäsion und Korrosion) kommt es zum Verschleiß des Schneckenstegs und des Massezylinders. Daraus resultiert eine Erhöhung des Spaltmaßes, was in weiterer Folge zu Leckströmungen entgegen der Förderrichtung führt und somit den Materialdurchsatz verringert. Zur Messung des Spaltmaßes wurde eine ultraschallbasierte Messmethode entwickelt die es erlaubt ohne langwierige Produktionsunterbrechungen den Verschleißstatus zu ermitteln. Das entwickelte System erlaubt es auch die Schließzeiten der sogenannten Rückstromsperre beim Spritzgießen zu messen. Die Rückstromsperre an der Schneckenspitze verhindert - beim Einspritzen des Kunststoffes in das Spritzgießwerkzeug - das Rückfliessen der Schmelze zurück in den Schneckenkanal. Eine Erhöhung der Schließdauer während des Produktionsbetriebes deutet auf einen Verschleiß einzelner Komponenten der Rückstromsperre hin. Das entwickelte System reduziert die Stillstandszeit der Spritzgießmaschine für die Verschleißermittlung von Stunden bzw. Tagen (in Abhängigkeit der Maschinengröße) auf wenige Minuten.
\end{abstract}

Keywords: Zustandsüberwachung, Verschleiß, Spritzgießen, Ultraschall

\section{Einleitung}

Beim Spritzgießen bzw. bei der Extrusion von Kunststoffen wird typischerweise der Rohstoff in Pulver- oder Granulatform in einem beheizten Massezylinder mittels einer rotierenden Schnecke plastifiziert und danach verfahrensabhängig weiterverarbeitet. Das Aufschmelzen des Kunststoffes erfolgt dabei einerseits durch Wärmeleitung (über den beheizten Massezylinder) und andererseits durch Friktion (durch die Rotation der Schnecke). Während des Prozesses werden die Stege der Plastifizierschnecke und die Innenwand des Massezylinders durch abrasive (Füllstoffe in der Kunststoffschmelze), adhäsive (Kontakt Steg/Zylinder) und korrosive Verschleißvorgänge (flüchtige Bestandteile in der Schmelze) abgenützt. Dieser Verschleiß führt zu einer Erhöhung des Spaltmaßes zwischen den beiden Komponenten und in weiterer Folge zu Leckströmungen entgegen der Förderrichtung.
Meist wird versucht mit höheren Drehzahlen den geringeren Massedurchsatz zu kompensieren was jedoch zu einer erhöhten Energieaufnahme führt und den Kunststoff durch die erhöhten Scherraten schädigen kann. Die übliche Vorgehensweise zur Ermittlung des Verschleißes ist der Ausbau der Schnecke, Reinigung der Schnecke und des Massezylinders von Kunststoffresten und der darauffolgenden Vermessung des Außendurchmessers der Schnecke bzw. des Innendurchmessers des Massezylinders. Dies führt zu teils tagelangen Unterbrechungen der Produktion und dementsprechend hohen Kosten. Es gab nur wenige Untersuchungen zu Messung des Verschleißes der Plastifiziereinheit während des laufenden Betriebes. Mittels Radionuklid-Technik [1] wurde der abrasive Verschleiß in einem Extruder gemessen. Aufgrund der aufwändigen Datenauswertung und des nötigen Strahlenschutzes (die zu untersuchenden Elemente müssen radioaktiv markiert werden) ist die Methode für den 
industriellen Einsatz wenig geeignet und auf demensprechend ausgestattete Labors beschränkt.

Mittels

Röntgenfluoreszenzanalyse (RFA) [2] wurde versucht Verschleißpartikel in der plastifizierten Schmelze eines Extruders zu detektieren. Die Anforderungen an den Strahlenschutz sind bei der RFA Methode zwar geringer, da keine radioaktive Aktivierung erfolgen muss, allerdings müssen für quantitative Messungen aufwändige Kalibrationen durchgeführt werden. Des Weitern kann mit dieser Methode nicht die Position des Verschleißes bestimmt werden. Mit Schallemissionsanalyse [3] wurde versucht die Anstreifvorgänge zwischen Schneckensteg und Zylinderinnenwand an einem Laborextruder zu detektieren und die akustische Emissionsrate mit dem adhäsiven Verschleiß zu korrelieren. Die Methode ist prinzipiell für den industriellen Einsatz geeignet, quantitativen Aussagen über die Position und Ausmaß des Verschleißes können jedoch damit nicht getroffen werden. In [4] wurden erstmals der Spalt zwischen Schnecke und Zylinder mittels nicht-invasiver Ultraschalltechnik ermittelt. Dazu wurde die Differenz der Pulslaufzeit zwischen Zylinderinnenwand und Schneckensteg analysiert. Die benötigte Schallgeschwindigkeit der Kunststoffschmelze im Spalt wurde in Kalibrationsmessungen ermittelt. In dieser Arbeit wird ein ultraschallbasiertes System vorgestellt, dass es ermöglicht durch den mindestens $200^{\circ} \mathrm{C}$ heißen Stahl des Massezylinders vollkommen automatisiert die Spaltweite zu ermitteln.

Die Bestimmung der Schließdauer der Rückstromsperre in Spritzgießmaschinen kann messtechnisch durch eine Druckmessung im Schneckenkanal hinter der Rückstromsperre realisiert werden. Beim Einspritzen des Kunststoffes in das Spritzgießwerkzeug steigt der Druck im Schneckenkanal hinter der Rückstromsperre an, bis die Sperre schließt und es zu einem Druckabfall kommt. Die Zeit vom Beginn des Druckanstieges bis zum Abfall entspricht der Schließdauer. Für eine derartige Messung werden Drucksensoren benötigt die den Drücken bis ca. 2000 bar standhalten und im Massezylinder dementsprechend eine Sensorbohrung vorgesehen werden. Die Messung der druckabhängigen Schalllaufzeit im Schneckenkanal hinter der Rückstromsperre - wie sie in dieser Arbeit vorgestellt wird - erlaubt die nicht-invasive Ermittlung der Schließdauer der Rückstromsperre und somit Rückschlüsse auf den Verschleißstatus.

\section{Systemmodell}

In Abb. 1 ist das tribomechanische System Schnecke/Zylinder schematisch dargestellt.
Werden an einer axial fixierten Position Ultraschallpulse in den Massezylinder gesendet kommt es an den verschiedenen Grenzflächen Kunststoff/Schneckensteg,

Kunststoff/Schneckengrund) zu Reflexionen die mit einem Ultraschallsensor detektiert werden können. Das Spaltmaß ist bestimmt durch

$$
\delta=\frac{z_{i}-s_{a}}{2} \pm a
$$

wobei $z_{i}$ den Innendurchmesser des Massezylinders, $s_{a}$ den Außendurchmesser der Schnecke und a die Auslenkung der Schnecke aus der Zentrumslage in Richtung des Ultraschallsensors bezeichnet. Diese Auslenkung kann statisch sein (Symmetrieachse Zylinder/Schnecke nicht ident), in den meisten Fällen wird jedoch eine dynamische Auslenkung der Schnecke beobachtet.

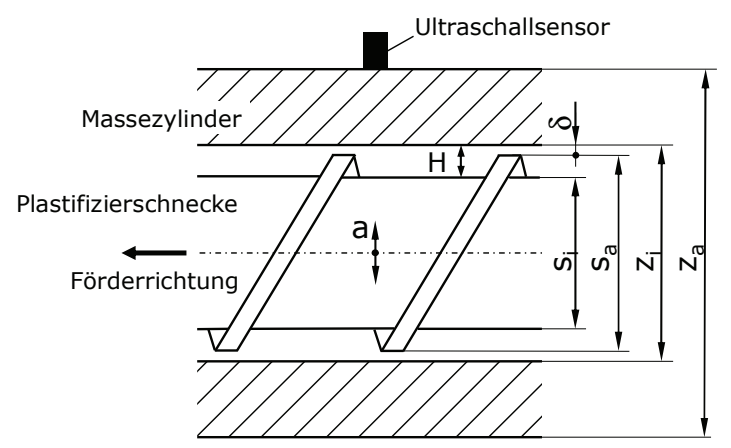

\section{Abb. 1: Schematische Darstellung des tribomechanischen Systems Massezylinder/Plastifizierschnecke}

Diese dynamische Exzentrizität wurde in [5] untersucht. Es wurde gezeigt, dass die Spaltweite an der Messposition alle 2 Umdrehungen konstant ist (two-cycle repetition phenomenon). Der Grund ist eine sinusförmige Auslenkung $a$ in Richtung des Ultraschallsensors, welche mit der exakt doppelten Periodendauer in Bezug auf die Schneckendrehzahl verläuft [6]. Erklärbar ist ein solches Verhalten damit, dass sich die Symmetrieachse der Schnecke kreisförmig um die Symmetrieachse des Massezylinders bewegt (vermutlich durch Druckgradienten entlang des Schneckenkanals die zu dieser periodischen Auslenkung führen). Weiters wurde in [6] gezeigt das eine Mittelung der sich periodisch veränderten Spaltweiten während der Plastifizierung in guter Näherung dem tatsächlich Spaltmaß entspricht.

Die Kanaltiefe des Schneckenkanals ergibt sich mit

$$
H=\frac{z_{i}-s_{i}}{2} \pm a
$$


wobei $s_{i}$ den Innendurchmesser der Schnecke bezeichnet. Abb. 2 zeigt die schematische Darstellung einer Messung an einem abgewickelten Schneckenkanal (entspricht einer Umdrehung der Schnecke). Es können zwei Arten von Reflexionen beobachtet werden. Die Reflexion der Zylinderinnenwand mit der Laufzeit

$$
t_{z}=\frac{\left(z_{a}-z_{i}\right)}{c_{z}},
$$

wobei $c_{z}$ die Schallgeschwindigkeit im Zylinderstahl und $z_{a}$ den Außendurchmesser des Massezylinders bezeichnet, ist durchgehend erkennbar.

Bei Messung im Schneckenkanal kommt es zu einer Reflexion am Schneckengrund mit der Laufzeit

$$
t_{s}=\frac{2 H}{c_{\text {Schmelze }}(T, P)},
$$

wobei $c_{\text {Schmelze }}(T, P)$ die temperatur- und druckabhängige Schallgeschwindigkeit der Kunststoffschmelze bezeichnet. Gelangt der Schneckensteg in den Messbereichs des Sensors kommt es dort zur Reflexion des Ultraschallpulses. Die Laufzeit im Spalt (mit der Spaltweite $\delta$ ) zwischen Zylinderinnenwand und Schneckensteg ergibt sich mit

$$
t_{\delta}=\frac{2 \delta}{c_{\text {Schmelze }}(T, P)}
$$

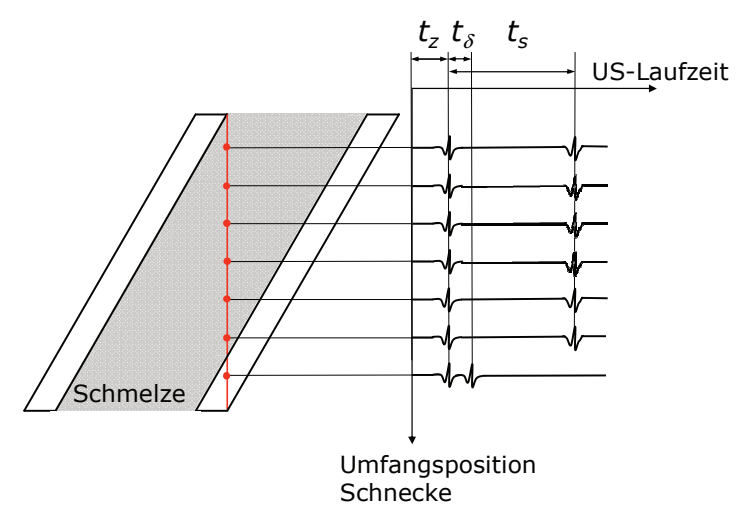

Abb. 2: Abgewickelter Schneckenkanal und Darstellung der verschiedenen Reflexionen während der Plastifizierung.

Wie aus Gl. (5) ersichtlich ist, muss für die Ermittlung der Spaltweite $\delta$ die Schallgeschwindigkeit in der Schmelze bekannt sein. $\mathrm{Da}$ der Schneckengrund und die Massenzylinderinnenwand in der Regel weniger vom Verschleiß betroffen sind als der Schneckensteg, wird die nominelle Kanaltiefe $H$ (die Auslenkung $a$ in $\mu \mathrm{m}$ Bereich ist hier vernachlässigbar) verwendet um die Schallgeschwindigkeit abzuschätzen. Da durch Temperatur- und Druckgradienten im Schneckenkanal die Laufzeit $t_{s}$ nicht konstant ist wird über alle Signale gemittelt:

$$
\bar{c}_{\text {Schmelze }}=\frac{1}{n_{s}} \sum_{n=1}^{n_{s}} \frac{2 H}{t_{s, n}},
$$

wobei $n_{s}$ die Gesamtanzahl der Messungen von Signalen mit einer vorhandenen Schneckengrundreflexion bezeichnet. Diese mittlere Schmelzetemperatur lässt sich nun dazu verwenden - zusammen mit den gemessenen Laufzeiten im Spalt - um den mittleren Abstand zwischen Zylinderinnenwand und Schneckensteg zu ermitteln:

$$
\bar{\delta}=\frac{1}{n_{\delta}} \sum_{n=1}^{n_{\delta}} \frac{\bar{c}_{\text {Schmelze }} t_{\delta, n}}{2}
$$

mit $n_{\delta}$ der Gesamtanzahl der Reflexionssignale vom Schneckensteg.

Die Funktionsweise einer Rückstromsperre ist in Abb. 3 dargestellt. Beim Plastifizieren rotiert die Schnecke und zieht sich dabei zurück. Der Sperrring liegt dabei auf den Flügeln der rotierenden Schneckenspitze. Aufgrund der hohen Relativgeschwindigkeit zwischen Schneckenspitze und dem sich freidrehenden Sperrrings kommt es an den Kontaktflächen zu einem Abtrag von Material (hauptsächlich an den Flügeln der Schneckenspitze).
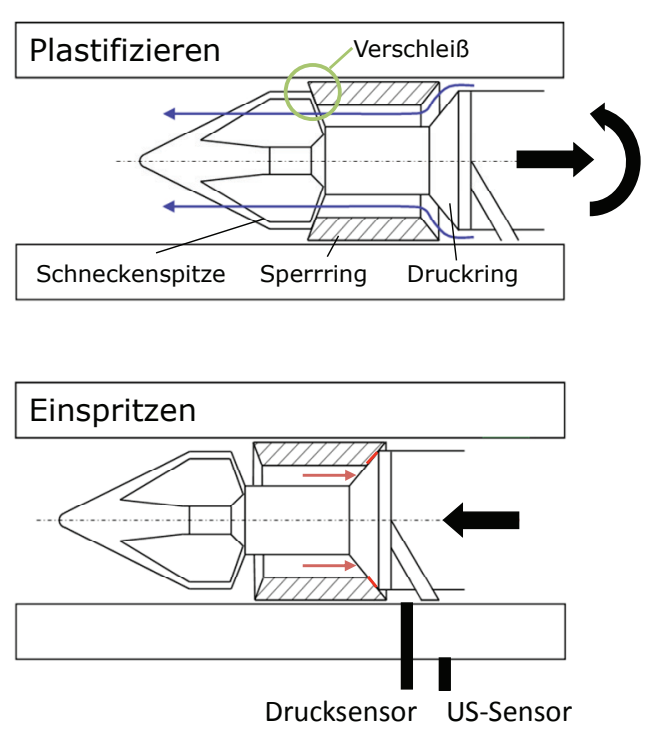

$\begin{array}{llr}\text { Abb. 3: } & \text { Funktionsweise } & \text { einer } \\ & \text { Rückstromsperre } & \text { und } \\ & \text { Verschleißzonen. } & \end{array}$

Beim Einspritzen der Schmelze vom Schneckenvorraum in das Spritzgießwerkzeug wird die Schnecke kolbenartig nach vorne bewegt. Aufgrund des Druckanstieges im Schneckenvorraum wird der Sperrring gegen 
den Druckring gepresst und verhindert das Rückströmen der Schmelze in den Schneckenkanal. Die Konstanz dieser Schließbewegung ist ein wichtiges Kriterium um Gewichtsschwankungen (und damit einhergehend z.B. Schwankungen mechanischer Eigenschaften) der zu produzierenden Formteile zu minimieren. Der Verschleiß der Flügel der Schneckenspitze führt zu längeren Schließwegen und höheren Schwankungen der Schließzeiten. Eine quantitative Messung der Schließzeiten ist mittels eines Drucksensors hinter der Rückstromsperre möglich. Beim Einspritzen steigt der Druck im Schneckenkanal an bis der Sperrring gegen den Druckring gepresst wird und es zu einem Druckabfall kommt, wobei der Zeitpunkt vom Start des Einspritzens bis zum Druckabfall der Schließdauer entspricht. Da derartige Druckfühlerbohrungen an Standardmaschinen in der Produktion nicht vorhanden sind, kann hier mittels Ultraschall die druckabhängige Schallgeschwindigkeit im Schneckenkanal hinter der Rückstromsperre ohne Modifikationen am Massezylinder während des Betriebes gemessen werden und daraus die Schließdauer und Funktionsfähigkeit der Rückstromsperre abgeleitet werden.

\section{Signalverarbeitung zur Ermittlung der Laufzeit $\boldsymbol{t}_{\delta}$}

Aufgrund der großen Differenz der akustischen Impedanzen des Zylinderstahls $z_{z}$ und der Kunststoffschmelze $z_{\text {Schmelze }}$ ist es nur möglich einen kleinen Teil, der vom Ultraschallsensor in den Zylinderstahl eingekoppelte Schallenergie über die Grenzfläche Zylinder/Schmelze zu übertragen. Der überhebliche Anteil wird an dieser Grenzfläche reflektiert. Der Reflexionskoeffizient errechnet sich mit

$$
R=\frac{z_{z}-z_{\text {Schmelze }}}{z_{z}+z_{\text {Schmelze }}}
$$

mit $z_{Z}=\rho_{Z} c_{Z}$ und $z_{\text {Schmelze }}=\rho_{\text {Schmelze }} c_{\text {Schmelze }}$. $\mathrm{Da}$ die Dichte des Stahls $\rho_{Z}$ und die Schallgeschwindigkeit im Zylinderstahl $C_{z}$ bekannt sind und die Dichte $\rho_{\text {Schmelze }}$ und Schallgeschwindigkeit $c_{\text {Schmelze }}$ gut abgeschätzt werden können, kann der Reflexionskoeffizient $R$ in guter Näherung bestimmt werden (Für Kunststoffschmelzen typischerweise im Bereich $R \sim 0.95$ - 0.98). Da das Signal $S_{\text {Zylinder }}$ der Hauptreflexion an dieser Grenzfläche die Signale der Reflexionen vom Schneckensteg $S_{\text {Spalt }}$ bzw. Schneckengrund $S_{\text {Grund }}$ deutlich überlagert, muss es durch eine sogenannte Mittelwertbildung aus den gemessenen Daten herausgerechnet werden: Es wird der Mittelwert über alle gemessenen Signale gebildet und danach von den einzelnen gemessenen Signalen subtrahiert. Während die Laufzeiten zum Schneckengrund relativ einfach durch Kreuzkorrelation des Signals $S_{\text {Zylinder }}$ mit den invertierten Signalen vom Schneckengrund ermittelbar sind, kommt es bei den Versuch die Signale vom Schneckensteg zu detektieren häufig zu Fehlbestimmungen der Laufzeit. An den schmalen Spalt (etwa $100 \mu \mathrm{m}$ ) kommt es zu sich überlagernden Mehrfachreflexionen welche die empfangene Signalform (im Vergleich zu $S_{\text {Zylinder }}$ ) deutlich verändern und somit die Laufzeit nicht mehr fehlerfrei mittels einer Kreuzkorrelation bestimmbar ist. In Abb. 4 sind die Zusammenhänge der Messung zwischen Zylinderinnenwand und Schneckensteg schematisch dargestellt.

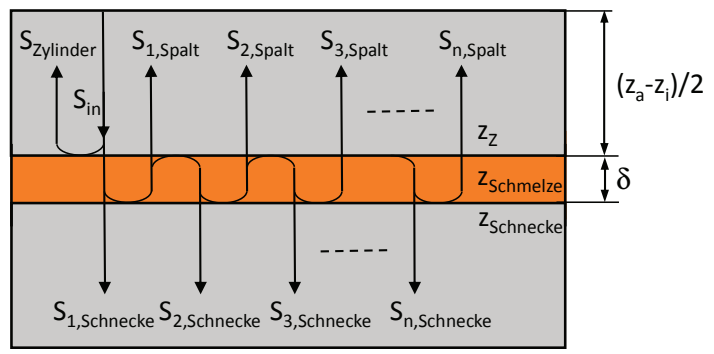

$\begin{array}{ll}\text { Abb. 4: } & \text { Schematische Darstellung der } \\ & \text { Signale bei der Messung am } \\ \text { Schneckensteg. } & \end{array}$

Der eingekoppelte Ultraschall wird an der Zylinderinnenwand reflektiert (resultierendes Signal $S_{Z y \text { linder }}$. Ein Teil der Schallenergie läuft durch die Kunststoffschmelze zum Schneckensteg wo ein Teil reflektiert wird und am Ultraschallsensor in das Signal $S_{1, \text { Spalt }}$ gewandelt werden kann (der transmittierte Anteil $S_{1, S c h n e c k e}$ gehen als Verluste in die Schnecke). Aufgrund der relativ geringen Schallabsorption, in dem mit Schmelze gefüllten Spalt, kommt es in weiterer Folge zu weiteren Reflexionen, deren resultierenden Signale sich gegenseitig überlagern. Das Signal der multiplen Reflexionen aus dem Spalt kann somit angeschrieben werden als

$$
S_{\text {Spalt }}(t)=\sum_{n} S_{n, \text { Spalt }}(t)
$$

wobei $S_{n, \text { Spalt }}(t)$ die $n$-te Reflexion am Schneckensteg bezeichnet. Das gemessene Signal kann beschrieben werden durch:

$$
S_{\text {Gemessen }}(t)=S_{\text {Zylinder }}(t)+S_{\text {Spalt }}(t)(10)
$$

Aufgrund der zeitdiskreten Messdatenverarbeitung (mit der Abtastzeit $T_{a}$ des Analog-Digital-Konverters) ergibt sich

$$
S_{\text {Gemessen }}[k]=\left.S_{\text {Gemessen }}(t)\right|_{t=k T a} \text {. }
$$

Die Spaltreflexion ergibt sich somit aus

$$
S_{\text {Spalt }}[k]=S_{\text {Gemessen }}[k]-S_{\text {Zylinder }}[k],
$$

wobei $S_{Z y l i n d e r}[k]$ durch die Messung im Bereich des Schneckengrundes (wo es aufgrund der 
relativ hohen Gangtiefe $H$ zu keiner Überlagerung der Signale kommt) bekannt ist. Zur Ermittlung der Laufzeit des Ultraschalls durch die Spaltbreite $\delta$, kann aus der Zylinderreflexion $S_{\text {Zylinder }}$ das Spaltsignal bestehend aus den multiplen Reflexionen mittels [7]

$$
S_{\text {sim }}[k]=-\left(\sum_{m=0}^{m_{\max }} S_{\text {Zylinder }}[k-m i] R^{2 m}\right)
$$

simuliert werden, wobei $m_{\max }$ die Anzahl der berücksichtigen Mehrfachreflexionen bezeichnet. Der Reflexionskoeffizient $R$ kann durch Gl. (8) abgeschätzt werden. Der Term $R^{2 m}$ beschreibt die Abschwächung der jeweiligen Reflexion im Spalt durch den fortlaufenden Kontakt mit den Grenzflächen Kunststoff/Zylinder bzw. Kunststoff/Schnecke. Eine Absorption des Schalls im Kunststoff wird aufgrund der geringen Spaltweite vernachlässigt, ebenso wie die Absorption im Zylinderstahl. Durch Variation der Abtastzeitpunkte von $i=1$ bis $i=i_{\max }$ (Schrittweite $i=i+1)$ können somit die multiplen Reflexionen bei verschiedenen Laufzeiten $t_{\delta}=i$ Ta simuliert werden. Dieses simulierte Signal $S_{\text {Sim }}[k]$ kann mittels linearen Korrelationskoeffizienten mit dem tatsächlichen

Spaltsignal $S_{\text {Spalt }}[k]$ verglichen werden. Durch Bildung eines Vektors mit den Korrelationskoeffizienten

$$
C[i]=\frac{\operatorname{Cov}\left(S_{\text {sim }}[k-i] S_{\text {Spalt }}[k]\right)}{\sqrt{\operatorname{Var}\left(S_{\text {sim }}[k-i]\right) \operatorname{Var}\left(S_{\text {Spalt }}[k]\right)}}(14)
$$

kann die tatsächliche Laufzeit im Spalt einfach bestimmt werden: Der resultierende Vektor $C$ besitzt ein Maximum beim Abtastzeitpunkt $i=i_{C \max }$ mit dem sich die tatsächlichen Laufzeit durch den Spalt mittels $t_{\delta}=i_{C \max } T_{a}$ bestimmen lässt.

\section{Systemaufbau}

Zur Messung (am bis $\mathrm{zu} 350^{\circ} \mathrm{C}$ heißen) Massezylinder wurde ein magnetisches Clamp-On System entwickelt, dass es erlaubt ohne Modifikationen am Zylinder die Ultraschallsonde an der gewünschten Messposition anzubringen. Abb. 5 zeigt eine Fotografie des entwickelten Systems: zwei Magnethalter sind an einem Verbindungsbügel drehbar gelagert. Die Ultraschallsonde kann orthogonal zur Symmetrieachse der Schnecke in eine Bohrung am Verbindungsbügel eingebracht werden. Die Sonde selbst besteht aus einer Piezokeramikscheibe (Mittenfrequenz $5 \mathrm{MHz}$ ) die auf einer kurzen Vorlaufstrecke verklebt ist und in einem Gehäuse verbaut wird. Zur Messung bei hohen Temperaturen kann die Sonde mit gekühlter Druckluft gespült werden. Die Anregung der Ultraschallsonde folgt über eine Pulser-
Receiver Einheit OPBOX 2.0 (Optel, Polen) mit einem $350 \mathrm{~V}$ Puls. Die empfangenen Signale werden mit dem integrierte Analog-DigitalKonverter (100 MHz Abtastfrequenz, 10-bit Auflösung) digitalisiert und über eine USB Schnittstelle an den Auswerterechner übermittelt.

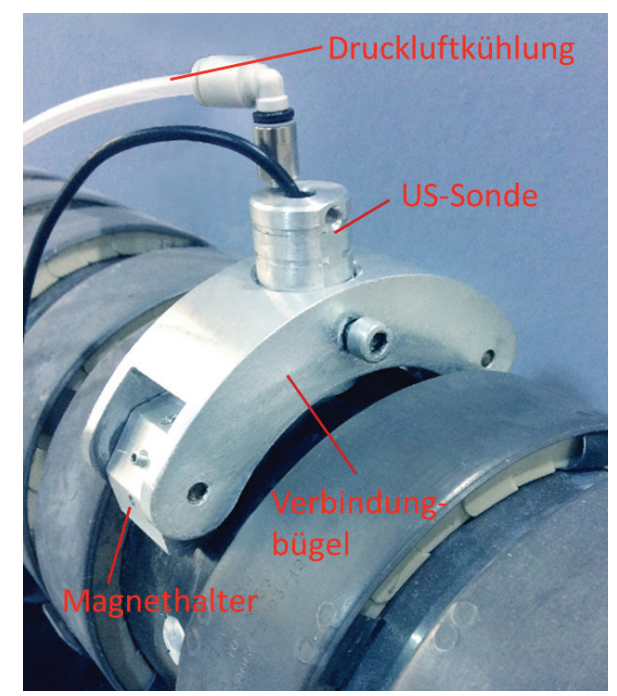

Abb. 5: Clamp-On System auf einem Massezylinder.

\section{Verifikationsmessungen}

Die Verifikation des entwickelten Algorithmus wurde an einer Standard-Dreizonenschnecke $(d=30 \mathrm{~mm})$ durchgeführt. Das tatsächliche Spaltmaß wurde konventionell (Ausbau der Schnecke, Vermessung der Komponenten) ermittelt.

Abb. 6 (a) zeigt die gemessenen Zylinderreflexion $S_{\text {Zylinder }}$ (gemessen im Bereich Schneckenkanals) und Abb. 6 (b) die Spaltreflexion $S_{\text {Spalt. }}$ Es ist deutlich erkennbar, dass sich die Signalformen deutlich (aufgrund der sich überlagernden Mehrfachreflexionen im Spalt) unterscheiden. Aus diesem Grund kann die Laufzeitbestimmung durch Kreuzkorrelation oder durch eine Bestimmung der Maxima der beiden Signale zu einer fehlerhaften Laufzeitbestimmung führen. Abb. 7 (a) zeigt den resultierenden Vektors $C$ [i] des vorgestellten Algorithmus ( $m_{\max }=7, R=0.98$ ). Das Maximum liegt bei $i_{C \max }=17$ Abtastpunkten. In Abb. 7 (b) ist ein Vergleich der beiden Signale $S_{\text {Zylinder }}$ und $S_{\text {Sim }}$ (berechnet mittels Gl. 13 und $i=i_{\text {Cmax }}$ ) dargestellt. Der Korrelationskoeffizient $C\left[i_{C \max }\right]=0.98$ zeigt die hohe Übereinstimmung des gemessenen und simulierten Spaltsignals. Bei einer Schallgeschwindigkeit von $c_{\text {Schmelze }}=950 \mathrm{~m} / \mathrm{s}$ und einer Abtastzeit $T_{a}=10 \mathrm{~ns}$ ergibt das eine Pulslaufzeit $t_{\delta}=170$ ns im Spalt bzw. einer Spaltbreite von $\delta=80.75 \mu \mathrm{m}$ (in guter Übereinstimmung mit dem Ergebnis der konventionellen Messung $\delta=83 \mu \mathrm{m})$. Um 
mögliche radiale Schneckenbewegung während der Plastifzierung zu kompensieren, wird bei der tatsächlichen Überprüfung über mehrere Schneckenumdrehungen gemessen und danach entsprechend Gl. 6 und Gl. 7 das Spaltmaß ermittelt.
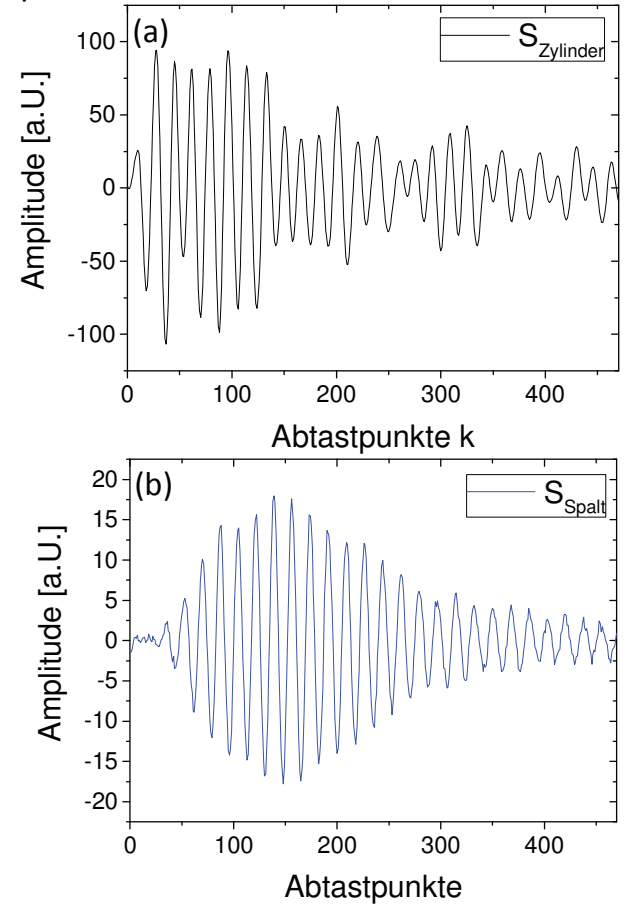

Abb. 6: (a) Reflexion der Zylinderinnenwand und (b) resultierendes Signal der multiplen Spaltreflexionen.
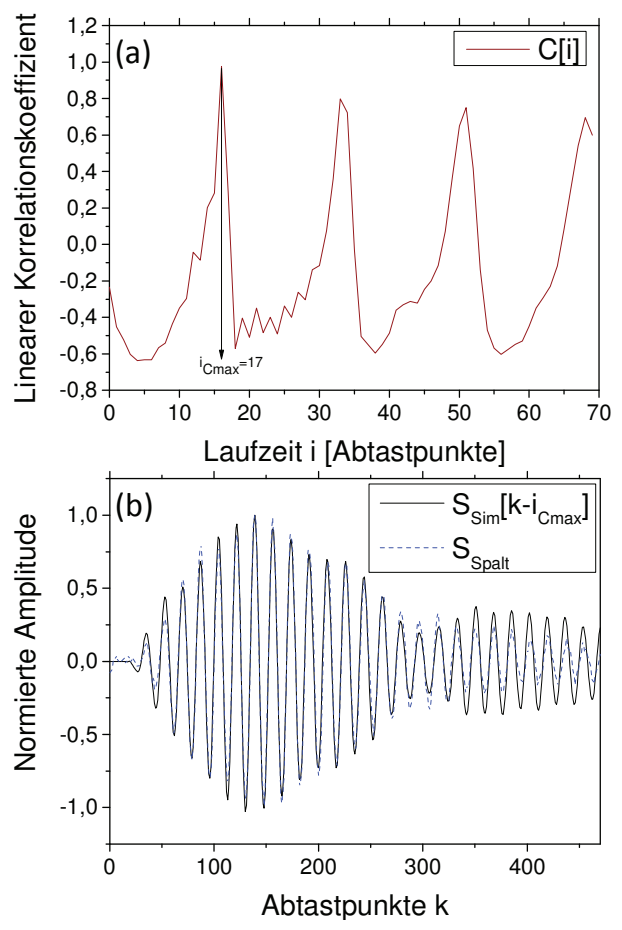

Abb. 7: (a) Lineare Korrelationskoeffizienten C[i] und (b) der Vergleich des gemessenen Spaltsignals $S_{\text {Spalt }}$ und des simulierten Spaltsignals $S_{\text {sim }}[k-$ $\left.i_{\text {Cmax }}\right]$

\section{Automatisierte Auswertung}

Abb. 8 zeigt das Ergebnis einer Messung während der Plastifizierung (Pulswiederholfrequenz $100 \quad \mathrm{~Hz}$, Schneckendurchmesser $60 \mathrm{~mm}$ ). Deutlich zu erkennen (nachdem die Zylinderinnenwandreflexion mittels Mittelwertbildung herausgerechnet wurde) sind die Reflexionen des Schneckensteges und des Schneckengrundes. Des Weiteren ist die Aufund Abwärtsbewegung des Schneckensteges aufgrund der dynamischen Exzentrizität der Schnecke erkennbar (two-cycle repetition phenomenon).

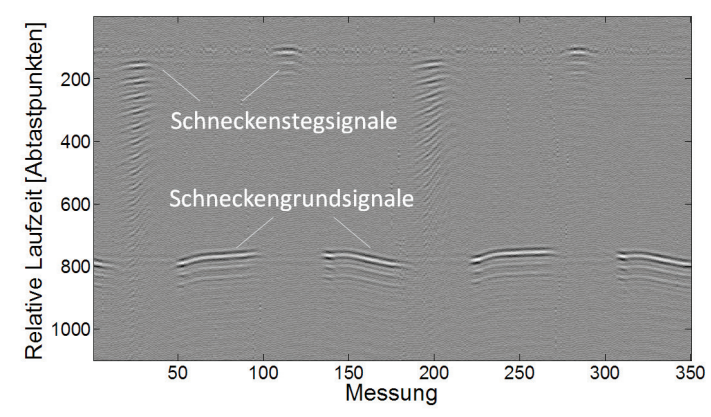

Abb. 8: Messergebnis bei Messung über mehrere Schneckenumdrehungen.

Das Ergebnis der automatisierten Auswertung ist in Abb. 9 dargestellt.

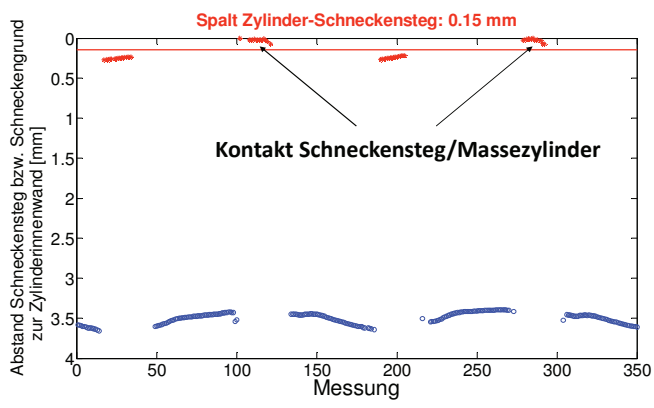

Abb. 9: Ergebnis der Schneckenspaltbestimmung bzw. der radialen Schneckenauslenkung.

Es ist zu erkennen, dass es unter den vorliegenden Prozessbedingungen zum Anstreifen des Schneckensteges an die Zylinderinnenwand kommt (was einen schnellen Verschleiß der Schnecke verursacht). Der ermittelte Mittelwert (durchgezogenen rote Linie, Abb. 9) für den Spalt zwischen Schneckensteg und Zylinderinnenwand beträgt $\delta=0.15 \mathrm{~mm}$ und ist in guter Übereinstimmung mit dem konventionell ermittelten Wert $(0.14 \mathrm{~mm})$. 


\section{Schließverhalten Rückstromsperre}

Neben der Verschleißmessung an der Plastifiziereinheit, kann mit dem ultraschallbasierten System auch das Schließverhalten der Rückstromsperre beurteilt werden. Abb. 10 zeigt die Pulslaufzeit im Schneckenkanal hinter der Rückstromsperre als Funktion der Einspritzdauer. Am Beginn des Einspritzens der Formmasse vom Schneckenvorraum in das Spritzgießwerkzeug kommt es zu einem Druckanstieg. Dieser Druckanstieg führt zu einer Verkürzung der Pulslaufzeit. Beim Schließen der Rückstromsperre (Schließdauer $t_{s}$ ) führt der abrupten Druckabfall im Schneckenkanal zu einer dementsprechenden Verringerung der Pulslaufzeit.

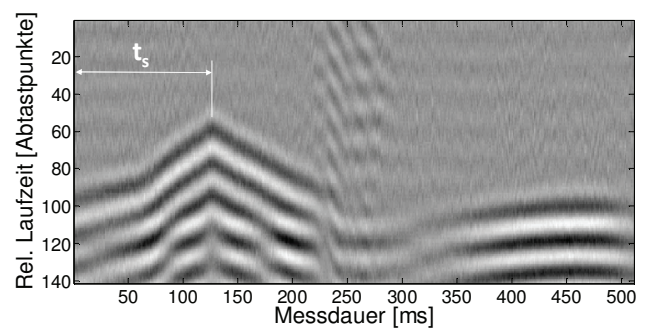

\section{Abb. 10: Pulslaufzeit als Funktion der Einspritzdauer.}

Mit derartigen Messungen kann die Konstanz der Schließbewegung der Rückstromsperre über mehrere Spritzgießzyklen bewertet werden bzw. ob die Rückstromsperre ordnungsgemäß schließt.

\section{Zusammenfassung}

In diesem Beitrag wurde ein ultraschalbasiertes System vorgestellt, das es erlaubt den Verschleißstatus des tribomechanischen Systems Plastifizierschnecke/Massezylinder und der Rückstromsperre abzuschätzen. Die benötigten Komponenten (Clamp-On System, Ultraschallsonde) und Algorithmen wurden unter praxisgerechten Bedingungen getestet und verifiziert. Das Messsystem erlaubt es Produktionsunterbrechungen zur Verschleißmessung von Stunden (bzw. Tagen) auf wenige Minuten zu reduzieren.

\section{Literaturnachweis}

[1] H. Schüle, H.G. Fritz, Entstehung und Minimierung des abrasiven Verschleißes bei Nutenextrudern, Kunststoffe 75, 399-403 (1985)

[2] R. Fox, Quantifizierung des Verschleißes kunststoffverarbeitender Maschinen mittels kontinuierlicher (zeitaufgelöster)

Röntgenfluoreszenzanalyse (RFA), Dissertation TU Darmstadt (2005)

[3] G. Paller, Theoretische und experimentelle Untersuchungen zum tribologischen System Schneckensteg/Zylinderwand in EinschneckenPlastifiziereinheiten, Dissertation TH Darmstadt (1993)

[4] C.-K.i Jen et al, Meas. Sci. Technol. 16, 842850 (2005); doi: 10.1088/0957-0233/16/3/028

[5] Z. Sun, L.A. Utracki, Polym. Eng. Sci.49, 244250 (2009); doi: 10.1002/pen.21246

[6] B. Praher, K.Straka, G. Steinbichler, Noninvasive Ultrasound Based Reflection Measurements at Polymer Plastication Units: Measurement of Melt Temperature, Melting Behaviour and Screw Wear, ITG- Fachbericht, Sensoren und Messsysteme (2014)

[7] B. Praher, G. Steinbichler, Ultrasound-based measurement of liquid-layer thickness: A novel time-domain approach, submitted to Mech. Syst. Signal. Pr. (2015) 